\section{Agrupamento da Classificação Internacional de Doenças para análise de reinternações hospitalares}

\author{
Grouping of the International Classification \\ of Diseases for analysis of hospital readmissions
}

\author{
1 Escola Nacional de Saúde \\ Pública, Fundação Oswaldo \\ Cruz, Rio de Janeiro, Brasil. \\ Correspondência \\ M. S. M. Castro \\ Escola Nacional \\ de Saúde Pública, \\ Fundação Oswaldo Cruz. \\ Rua Engenheiro Amaro \\ Lanari 355, apto. 601, \\ Belo Horizonte, $M G$ \\ 30310-580, Brasil. \\ mmcastro@uai.com.br
}

\begin{abstract}
ICD chapters and three-digit categories are not always appropriate for epidemiological analysis; the former are too generic and the latter too numerous. When the epidemiological focus is not on a specific clinical diagnosis, an intermediate grouping proposal between these two possibilities becomes necessary. Such proposals are not frequent in the literature. We used a twostage grouping proposal based on hospital admissions volume, internal clinical coherence in each group, and the results of a Cox multivariate model for real data. As a result, we obtained 26 groups for adults and 19 groups for children, among which we chose hernia as the reference category for adults and appendiceal disease for children. The grouping proved adequate for the analysis of readmissions in a large Brazilian general hospital. Procedures similar to those described in this research report can be used to analyze other health problems.
\end{abstract}

International Classification of Diseases; Hospital Readmission; Diagnosis
Mônica S. Monteiro de Castro 1

Marilia Sá Carvalho 1

\section{Introdução}

A inclusão da informação diagnóstica presente nas Autorizações de Internação Hospitalar (AIH) em modelos multivariados representa um desafio para os pesquisadores. Neste artigo, propomos uma forma de agrupamento de diagnósticos da AIH para análise de dados de reinternação hospitalar.

Os diagnósticos das AIH são codificados utilizando-se a Classificação Internacional de Doenças (CID), proposta pela Organização Mundial da Saúde (OMS), atualmente na Décima Revisão (CID-10) 1. Por um lado, os capítulos da CID-10 e da CID-9 (Classificação Internacional de Doenças - Nona Revisão) 2 englobam um número muito grande de patologias que diferem entre si quanto ao risco da internação hospitalar. Por outro lado, as categorias de três dígitos da CID-9 e da CID-10 são excessivamente numerosas para que se possa incluí-las como variável categórica na análise. Por essas razões, quando o interesse não recai sobre um grupo diagnóstico específico, formas alternativas de agrupamento de categorias de três dígitos se fazem necessárias.

As listas de tabulação para morbidade habitualmente propostas para a CID pela OMS ainda possuem um número muito grande de categorias. Por exemplo, as listas que serviram de ponto de base para o nosso estudo possuíam 141 categorias para a CID-9 e 234 para a CID- 
10, número ainda excessivo para inclusão em modelos multivariados. O nosso interesse, então, foi obter uma lista própria alternativa a esse excesso de categorias que fosse de tamanho adequado para uso em modelos estatísticos.

Uma dificuldade para analisar informações de AIH incluindo períodos de tempo anteriores a 1999 é a necessidade de trabalhar com duas classificações simultaneamente. O Ministério da Saúde determinou que, a partir de janeiro de 1999, as AIH deveriam ser preenchidas utilizando os códigos da CID-10. Até então, era utilizada a CID-9. Apesar das dificuldades para compatibilização das duas classificações, essa mudança foi benéfica, pois a CID-10 apresenta vantagens em relação a CID-9 3,4.

Este artigo apresenta uma proposta de agrupamento intermediária entre as categorias de três dígitos e os capítulos, desenvolvida especialmente para analisar reinternações hospitalares em um grande hospital geral brasileiro, englobando internações codificadas pela CID-9 e pela CID-10. O nosso objetivo é que a metodologia proposta abra caminhos para o tratamento da informação diagnóstica da CID, entendendo sempre que tabulações e agrupamentos devem servir ao objetivo da investigação.

\section{Metodologia}

Nós começamos pela lista de grupos de categorias de três dígitos da CID-9 e da CID-10, com 141 e 234 categorias, respectivamente, baseadas nas listas disponíveis no CD-ROM de Movimento de Autorização Hospitalar 5,6.

Na primeira etapa, pelas causas de internação para adultos (maiores de 14 anos), fizemos uma subdivisão dos capítulos valendo-nos do agrupamento de categorias de três dígitos, respeitando a numeração dos capítulos da CID-9 (para a descrição completa do agrupamento, ver Tabela 1). Esse agrupamento foi feito de forma empírica pelas duas autoras em conjunto, tendo como meta principal manter a coerência clínica dentro de um mesmo capítulo da CID e um volume mínimo de internações em cada novo grupo.

Nesta etapa não houve um limite rígido para o número de internações em cada grupo, apenas cuidou-se para não criar grupos muito pequenos. Coerência clínica foi entendida como grupos de doenças que tivessem comportamento similar no que se refere ao risco de reinternação. Exemplificando, as doenças do aparelho circulatório apresentaram um grande volume de internações e nós optamos por dividir esse grupo em doenças do aparelho circula- tório, menos veias e linfáticos, e doenças das veias e linfáticos, por entender que esses dois grupos têm riscos diferentes de reinternação. Raciocínio semelhante foi feito para outras situações como, por exemplo, para as doenças do aparelho respiratório e digestivo. Os capítulos da CID foram mantidos separados nesta etapa, independentemente do número de internações registradas. A mesma estrutura foi aplicada para as causas de internação para crianças (menores de 14 anos). Resumindo, os critérios utilizados nesta primeira etapa de redução dos grupos de categorias foram coerência clínica quanto ao risco de reinternações dentro de um mesmo capítulo da CID e a garantia de um volume mínimo de internações em cada novo grupo formado.

Na segunda etapa, a partir do ajuste de modelos de riscos proporcionais de Cox 7 somente até a primeira reinternação para adultos e crianças em separado, alguns desses grupos de categorias de três dígitos foram agregados da seguinte forma: os grupos com freqüência menor que cem foram agregados a outro grupo do mesmo capítulo da CID, caso a razão de riscos (hazard ratio - $\mathrm{HR}$ ) estimada pelo modelo de Cox tivesse mesma direção e magnitude similar. Caso contrário, se o HR diferisse de forma importante dos outros grupos daquele capítulo da CID em magnitude ou direção ou se fosse não significativo, este grupo passou a fazer parte de um grupo chamado de outros, que incluía ainda os capítulos da CID sintomas, sinais e afecções mal definidas e contato com os serviços de saúde. Resumindo, os critérios nesta segunda etapa de redução dos grupos de categorias foram garantia de um volume de internações em cada grupo formado (no mínimo cem internações), a coerência clínica e os resultados do ajuste do modelo de Cox para os dados reais a serem analisados. A escolha do modelo de Cox justifica-se pela estrutura dos dados em questão, já que a variável de interesse do estudo é o tempo até a reinternação.

O próximo passo necessário e extremamente importante nessa análise foi escolher o grupo que serviu como linha de base na análise multivariada, escolha essa que foi feita com base nos objetivos do estudo. Escolhemos como referência as doenças do apêndice para adultos e as hérnias para crianças. Após discussões com especialistas, tais grupos foram considerados como de menor risco de reinternação em geral, o que acabou comprovado pelos resultados dos modelos utilizando a classificação proposta 8 . 
Detalhamento do agrupamento de categorias da CID-9 e da CID-10 para análise dos dados de reinternação hospitalar.

\begin{tabular}{|c|c|c|c|c|c|}
\hline Capítulo & Nome do novo grupo & Grupos CID-9* & Código CID-9 & Grupos CID-10* & Código CID-10 \\
\hline I & Doenças infecciosas e parasitárias & 1 a $15+$ parte do 30 & 001 a 139 e 279 & 1 a 20 & $\mathrm{~A} 00$ a $\mathrm{B} 97$ \\
\hline II & Neoplasias & 16 a 26 & 140 a 239 & 21 a 37 & $\mathrm{C} 00$ a D48 \\
\hline IV & $\begin{array}{l}\text { Sangue, órgãos hematopoiéticos } \\
\text { e transtornos imunitários }\end{array}$ & 31 & 280 a 289 & 38 a 43 & D50 a D89 \\
\hline IIIA & Doenças endócrinas & 27 a 28 & 240 a 259 & 44 a 47 & E00 a E35 \\
\hline IIIB & Doenças nutricionais e metabólicas & 29 a 30 & 260 a 278 & 48 a 51 & E40 a E90 \\
\hline $\mathrm{V}$ & Doenças mentais & 32 a 35 & 290 a 319 & 52 a 62 & F00 a F99 \\
\hline VIA & Sistema nervoso & 36 a 39 & 320 а 359 & 63 a 73 & G00 a G99 \\
\hline VIB & Olhos e anexos, ouvido e apófise mastóide & 40 a 41 & 360 a 389 & 74 a 88 & $\mathrm{H} 00$ a H95 \\
\hline VIIA & Circulatório, menos veias e linfáticos & 42 a 49 & 390 a 448 & 89 a 96 e 98 & 100 a 179 e 195 a 199 \\
\hline VIIB & Veias e linfáticos & 50 & 451 a 459 & 97 & 180 a 189 \\
\hline VIIIA & Infecções respiratórias agudas & 51 e 53 & $\begin{array}{l}460 \text { a } 466 \text { e } 480 \\
\text { a } 487\end{array}$ & 99 a 101 & J00 a J22 \\
\hline VIIIB & Outras doenças respiratórias & 52 e 55 a 56 & $\begin{array}{l}470 \text { a } 478 \text { e } 500 \\
\text { a } 519\end{array}$ & 102 e 104 a 108 & $\begin{array}{l}J 30 \text { a J39 e J60 } \\
\text { a J99 }\end{array}$ \\
\hline VIIIC & Doenças crônicas das vias aéreas inferiores & 54 & 490 a 496 & 103 & $\mathrm{~J} 40$ a J47 \\
\hline IXA & Doenças do esôfago, estômago e duodeno & 58 & 530 a 537 & 110 & $\mathrm{~K} 20$ a K31 \\
\hline IXB & Doenças do apêndice & 59 & 540 a 543 & 111 & $\mathrm{~K} 35$ a K38 \\
\hline IXC & Hérnias da cavidade abdominal & 60 & 550 a 553 & 112 & $\mathrm{~K} 40$ a K46 \\
\hline IXD & Doenças dos intestinos e peritôneo & 61 a 62 & 555 a 569 & 113 a 115 & $\mathrm{~K} 50$ a K67 \\
\hline IXE & Outras doenças do aparelho digestivo & $57+$ parte do 63 & $\begin{array}{l}520 \text { a } 529 \text { e } 578 \\
\text { a } 579\end{array}$ & 109 e 118 & $\begin{array}{l}\text { K00 a K14 e K90 } \\
\text { a K93 }\end{array}$ \\
\hline IXF & Doenças do fígado & parte do 63 & 570 a 573 & 116 & K70 a K77 \\
\hline IXG & Vesícula biliar, vias biliares e pâncreas & parte do 63 & 574 a 577 & 117 & $\mathrm{~K} 80$ a K87 \\
\hline XII & Pele e tecido subcutâneo & 75 a 77 & 680 a 709 & 119 a 126 & L00 a L99 \\
\hline XIII & Osteomuscular e tecido conjuntivo & 78 a 81 & 710 a 739 & 127 a 132 & M00 a M99 \\
\hline$X A$ & Doenças urinárias & parte do $64+65$ & $\begin{array}{l}580 \text { a } 583 \text { e } 587 \\
\text { a } 599\end{array}$ & $\begin{array}{l}133,134,136 \\
\text { a } 138 \text { e } 143\end{array}$ & $\begin{array}{l}\text { N00 a N16, N20 } \\
\text { a N39 e N99 }\end{array}$ \\
\hline XB & Doenças genitais masculinas & 66 & 600 a 608 & 139 & N40 a N51 \\
\hline$x C$ & Doenças genitais femininas & 67 a 69 & 610 a 629 & 140 a 142 & N60 a N98 \\
\hline$X D$ & Insuficiência renal & parte do 64 & 584 a 586 & 135 & N17 a N19 \\
\hline $\mathrm{XV}$ & Afecções originadas no período perinatal & 83 & 760 a 779 & 152 a 161 & P00 a P96 \\
\hline$X I$ & Gravidez, parto e puerpério & 70 a 74 & 630 a 676 & 144 a 151 & О00 а 099 \\
\hline XIV & Malformações congênitas & 82 & 740 a 759 & 162 a 172 & Q00 a Q99 \\
\hline $\mathrm{XVI}$ & Sintomas, sinais e afecções mal definidas & 84 a 86 & 780 a 799 & 173 a 185 & R00 a R99 \\
\hline $\mathrm{XVII}$ & Lesões, envenenamentos e causas externas & 87 a 133 & $\begin{array}{l}800 \text { a } 999 \text { e E800 } \\
\text { a E999 }\end{array}$ & 186 a 227 & S00 a Y98 \\
\hline$X X I$ & Contato com os serviços de saúde & 134 a 141 & V01 a V82 & 228 A 234 & Z00 a Z99 \\
\hline
\end{tabular}

* Cada grupo representa uma categoria da lista de tabulação de morbidade que serviu de ponto de partida.

\section{Resultados}

Foram utilizadas nessa análise as distribui ções de freqüência da causa principal das primeiras internações de cada indivíduo ocorridas no Hospital Público Regional de Betim (HPRB), localizado em Betim, região metropolitana de Belo Horizonte, Minas Gerais, Brasil. Os dados analisados foram provenientes do Sistema de Informações Hospitalares do SUS (SIH-AIH) e correspondiam ao período de ju- lho de 1996 a junho de 2000, sendo 6.409 internações com código CID-9 e 21.237 com código CID-10.

A primeira etapa resultou na criação de 33 grupos (Tabela 2). Nesta tabela são mostrados os tamanhos de cada grupo e os resultados da análise multivariada que foram utilizados para a etapa seguinte. Como referência para o leitor, são apresentados também os capítulos da CID dos quais os grupos constituem subdivisões, utilizando nomenclatura de capítulos da 
Agrupamento de categorias da CID resultando em 33 grupos, para análise de dados de reinternação hospitalar.

\begin{tabular}{|c|c|c|c|c|c|c|c|c|c|}
\hline \multirow[b]{2}{*}{ Infecciosas e parasitárias } & \multirow[t]{2}{*}{ Capítulo } & \multicolumn{5}{|c|}{ Adultos } & \multicolumn{3}{|c|}{ Crianças } \\
\hline & & $\mathrm{n}$ & $\mathrm{RR}$ & LS IC95\% & LI IC95\% & $\mathrm{n}$ & $\mathrm{RR}$ & LS IC95\% & LI IC95\% \\
\hline Infecciosas e parasitárias & 1 & 444 & 1,00 & & & 1.086 & 1,00 & & \\
\hline Neoplasias & II & 415 & 1,24 & 0,91 & 1,70 & 34 & 1,14 & 0,41 & 3,18 \\
\hline Endócrinas & IIIA & 481 & 1,34 & 1,02 & 1,76 & 40 & 4,13 & 2,14 & 7,96 \\
\hline Nutricionais e metabólicas & IIIB & 90 & 1,36 & 0,86 & 2,15 & 71 & 0,85 & 0,38 & 1,94 \\
\hline $\begin{array}{l}\text { Sangue, órgãos hematopoiéticos } \\
\text { e transtornos imunitários }\end{array}$ & IV & 196 & 1,21 & 0,85 & 1,72 & 128 & 2,80 & 1,85 & 4,23 \\
\hline Mentais e comportamentais & V & 20 & 0,81 & 0,30 & 2,22 & 0 & NA & NA & NA \\
\hline Sistema nervoso & VIA & 1.095 & 1,06 & 0,83 & 1,36 & 283 & 2,53 & 1,78 & 3,61 \\
\hline $\begin{array}{l}\text { Olho e anexos, ouvido } \\
\text { e apófise mastóide }\end{array}$ & VIB & 46 & 0,46 & 0,11 & 1,87 & 99 & 1,69 & 0,91 & 3,15 \\
\hline $\begin{array}{l}\text { Circulatório, menos veias } \\
\text { e linfáticos }\end{array}$ & VIIA & 2.915 & 1,29 & 1,04 & 1,61 & 87 & 1,86 & 1,08 & 3,20 \\
\hline Veias e linfáticos & VIIB & 536 & 1,37 & 1,02 & 1,83 & 14 & 3,86 & 1,20 & 12,40 \\
\hline Infecções respiratórias agudas & VIIIA & 607 & 1,02 & 0,78 & 1,33 & 1.649 & 1,27 & 1,01 & 1,60 \\
\hline Outras doenças respiratórias & VIIIB & 306 & 1,28 & 0,89 & 1,83 & 348 & 0,54 & 0,30 & 0,98 \\
\hline $\begin{array}{l}\text { Doenças crônicas vias aéreas } \\
\text { inferiores }\end{array}$ & VIIIC & 625 & 1,51 & 1,18 & 1,94 & 652 & 1,68 & 1,27 & 2,23 \\
\hline Esôfago, estômago e duodeno & IXA & 263 & 1,16 & 0,83 & 1,63 & 18 & 3,85 & 1,42 & 10,40 \\
\hline Doenças do apêndice & IXB & 814 & 0,82 & 0,59 & 1,14 & 206 & 1,31 & 0,72 & 2,38 \\
\hline Hérnias & IXC & 766 & 1,02 & 0,74 & 1,40 & 357 & 0,63 & 0,36 & 1,08 \\
\hline Doenças do intestino e peritôneo & IXD & 301 & 1,47 & 1,06 & 2,06 & 137 & 0,99 & 0,54 & 1,80 \\
\hline $\begin{array}{l}\text { Outras doenças do aparelho } \\
\text { digestivo }\end{array}$ & IXE & 444 & 1,38 & 1,05 & 1,83 & 35 & 2,80 & 1,29 & 6,05 \\
\hline Fígado & IXF & 193 & 1,92 & 1,40 & 2,63 & 5 & 0,04 & 0,00 & 284000,00 \\
\hline $\begin{array}{l}\text { Vesícula biliar, vias biliares } \\
\text { e pâncreas }\end{array}$ & IXG & 896 & 1,54 & 1,19 & 2,01 & 12 & 2,22 & 0,54 & 9,13 \\
\hline Doenças urinárias & XA & 650 & 1,25 & 0,96 & 1,62 & 274 & 1,14 & 0,72 & 1,82 \\
\hline Doenças genitais masculinas & XB & 175 & 0,79 & 0,45 & 1,40 & 115 & 1,30 & 0,69 & 2,46 \\
\hline Doenças genitais femininas & XC & 742 & 1,09 & 0,81 & 1,48 & 4 & NA & NA & NA \\
\hline Insuficiência renal & $X D$ & 348 & 3,32 & 2,53 & 4,35 & 21 & 7,58 & 3,31 & 17,40 \\
\hline Gravidez, parto e puerpério & $X I$ & 1.337 & 3,00 & 2,32 & 3,88 & 13 & 0,04 & 0,00 & 200000,00 \\
\hline Pele e tecido subcutâneo & XII & 459 & 1,71 & 1,28 & 2,29 & 291 & 0,68 & 0,41 & 1,13 \\
\hline Osteomuscular e tecido conjuntivo & $X I I I$ & 453 & 1,15 & 0,83 & 1,59 & 149 & 1,10 & 0,60 & 2,01 \\
\hline Anomalias congênitas & XIV & 30 & 1,21 & 0,44 & 3,32 & 218 & 2,24 & 1,51 & 3,31 \\
\hline $\begin{array}{l}\text { Afecções originadas no período } \\
\text { perinatal }\end{array}$ & $x V$ & 25 & 2,73 & 1,53 & 4,85 & 2.293 & 0,61 & 0,48 & 0,78 \\
\hline $\begin{array}{l}\text { Sintomas, sinais e afecções } \\
\text { mal definidas }\end{array}$ & $X V I$ & 651 & 0,94 & 0,71 & 1,24 & 309 & 0,92 & 0,58 & 1,46 \\
\hline Causas externas & $\mathrm{XVII}$ & 4.747 & 0,82 & 0,65 & 1,04 & 1.236 & 0,64 & 0,43 & 0,97 \\
\hline Contato com os serviços de saúde & $X X I$ & 288 & 0,76 & 0,47 & 1,24 & 27 & 0,56 & 0,08 & 4,01 \\
\hline Ignorado & $\lg n$ & 58 & 0,66 & 0,32 & 1,36 & 21 & 0,81 & 0,20 & 3,26 \\
\hline
\end{tabular}

$\mathrm{RR}=$ rico relativo; $\mathrm{LS}=$ limite superior $\mathrm{LI}=$ limite inferior

CID-9 (I a XVII), mais o capítulo XXI da CID-10. Por exemplo, o capítulo de doenças do aparelho digestivo (IX) ficou dividido em 7 grupos (IXA a IXG).

A segunda etapa resultou em 26 grupos de diagnóstico para adultos (Tabela 3) e 19 grupos para crianças (Tabela 4), usados como variável categórica nos modelos de reinternação hospi- talar. Para adultos, o número de grupos resultantes foi ligeiramente maior que o número de capítulos da CID, embora organizados de forma bastante diferente dos capítulos, como era o nosso objetivo. Para crianças, o número de grupos resultantes foi ligeiramente menor que o número de capítulos da CID, neste caso mantendo uma organização mais próxima dos ca- 
pítulos da CID, fato que poderia ser explicado pelo menor número de internações analisadas para crianças e pela menor diversificação das causas de internações em crianças.

A Tabela 1, já comentada, apresenta o detalhamento do agrupamento de categorias da CID-9 e da CID-10 que foi realizado na primeira etapa, e os grupos se referem a categorias das listas de tabulação para morbidade que serviram de ponto de partida. Fundamentados neste detalhamento, os leitores interessados podem aplicar o formato descrito em seus próprios dados ou mesmo alterar os agrupamentos para atender objetivos específicos de uma dada pesquisa.

\section{Discussão}

A CID iniciou-se e permaneceu por muito tempo como uma classificação de causas de morte, mas tanto a nona quanto a décima revisão foram bastante expandidas com o intuito de possibilitar o seu uso em estatísticas de morbidade ${ }^{3}$. A nona e a décima revisão da CID têm sido tradicionalmente utilizadas em estudos de morbidade, por serem classificações de caráter universal, oferecendo possibilidade de classificar todos os agravos à saúde e permitindo comparações.

Vários temas de pesquisa relativos aos serviços de saúde, como por exemplo as reinternações hospitalares, precisam incorporar a informação do diagnóstico, normalmente codificado por intermédio da CID, em suas análises. Motivados pelo interesse em encontrar uma forma de incorporação do diagnóstico mais representativa do problema das reinternações do que os grandes capítulos da CID, nós partimos dos próprios dados e especificamos melhor a classificação do diagnóstico, baseando-nos em coerência clínica, volume de internações e resultados obtidos com o ajuste de modelo multivariado para dados reais. O processo pode ser útil à análise de outros dados de internação ou reinternação hospitalar, ou mesmo de outros problemas de saúde, e ele também pode ser adaptado para outros objetivos específicos de pesquisa.

Do ponto de vista prático, o processo empírico (ad hoc) desenvolvido por nós se mostrou adequado à finalidade para a qual ele foi proposto, a saber, incluir a informação do diagnóstico em modelos explicativos de reinternações hospitalares, como pode ser visto em Castro et al. 8. Em etapa anterior do trabalho, as autoras tinham utilizado inicialmente os capítulos da CID e concluíram que a mudança foi benéfica, no tocante a permitir a discussão

\begin{tabular}{|c|c|}
\hline \multicolumn{2}{|c|}{$\begin{array}{l}\text { Vinte e seis grupos de CID utilizados para análise de reinternação } \\
\text { hospitalar em adultos. }\end{array}$} \\
\hline $\begin{array}{l}\text { Grupos da } \\
\text { CID capítulos }\end{array}$ & Doenças do apêndice (IXB - referência) \\
\hline I & Infecciosas e parasitárias \\
\hline II & Neoplasias \\
\hline III & Endócrinas, nutricionais e metabólicas \\
\hline IV & Sangue, órgãos hematopoiéticos e transtornos imunitários \\
\hline IXA & Esôfago, estômago e duodeno \\
\hline IXC & Hérnias \\
\hline IXD & Doenças do intestino e peritôneo \\
\hline IXE & Outras doenças do aparelho digestivo \\
\hline IXF & Fígado \\
\hline IXG & Vesícula biliar, vias biliares e pâncreas \\
\hline VIA & Sistema nervoso \\
\hline VIIA & Circulatório, menos veias e linfáticos \\
\hline VIIB & Veias e linfáticos \\
\hline VIIIA & Infecções respiratórias agudas \\
\hline VIIIB & Outras doenças respiratórias \\
\hline VIIIC & Doenças crônicas vias aéreas inferiores \\
\hline$X A$ & Doenças urinárias \\
\hline $\mathrm{XB}$ & Doenças genitais masculinas \\
\hline$X C$ & Doenças genitais femininas \\
\hline$X D$ & Insuficiência renal \\
\hline$X I$ & Gravidez, parto e puerpério \\
\hline $\mathrm{XII}$ & Pele e tecido subcutâneo \\
\hline $\mathrm{XIII}$ & Osteomuscular e tecido conjuntivo \\
\hline XVII & Causas externas \\
\hline$X X I I$ & Outros* \\
\hline
\end{tabular}

* Outros em adultos engloba transtornos mentais e comportamentais, doenças dos olhos e anexos e ouvido e apófise mastóide, anomalias congênitas afecções originadas no período neonatal, sintomas, sinais e afecções mal definidas, contato com os serviços de saúde e ignorado.

mais específica dos grupos sujeitos a um maior risco de reinternação.

O processo de agrupamento proposto apresenta alguns desafios para o seu uso mais disseminado. Em primeiro lugar, o grande volu me de dados a serem manipulados e analisados exigiu a utilização de programas estatísticos de uso menos comum, como o SAS e o R 9 . Em segundo lugar, agrupamentos propostos para problemas diferentes certamente serão diferentes, de forma que as comparações entre estudos diversos não poderão ser feitas de modo direto. Em terceiro lugar, um mesmo agrupamento possibilita diferentes escolhas de categorias de referência, o que influencia diretamente nos valores obtidos para os parâmetros estimados. Nós reconhecemos que o processo utilizado é sujeito a imprecisões. Dada a falta de soluções práticas para esse pro- 
Tabela 4

Dezenove grupos de CID utilizados para análise de reinternação hospitalar em crianças.

\begin{tabular}{ll}
\hline CID capítulos & Hérnias (IXC - referência) \\
\hline IV & Infecciosas e Parasitárias \\
IXB & Sangue, órgãos hematopoiéticos e transtornos imunitários \\
IXD & Doenças do apêndice \\
VIA & Doenças do intestino e peritôneo \\
VIB & Sistema nervoso \\
VII & Olho e anexos, ouvido e apófise mastóide \\
VIIIA & Circulatório \\
VIIIB & Infecções respiratórias agudas \\
VIIIC & Outras doenças respiratórias \\
XA & Doenças crônicas vias aéreas inferiores \\
XB & Doenças urinárias \\
XII & Doenças genitais masculinas \\
XIII & Pele e tecido subcutâneo \\
XIV & Osteomuscular e tecido conjuntivo \\
XV & Anomalias congênitas \\
XVII & Afecções originadas no período perinatal \\
XXII & Causas externas \\
\hline IIIt & Outros* \\
\hline
\end{tabular}

* Outros em crianças engloba neoplasias, doenças endócrinas, nutricionais e metabólicas, doenças do esôfago, estômago e duodeno, fígado, vesícula biliar, vias biliares e pâncreas, outras doenças do aparelho digestivo, doenças genitais femininas, insuficiência renal, gravidez, parto e puerpério, sintomas, sinais e afecções mal definidas, contato com os serviços de saúde e ignorado.

blema e a necessidade de avaliar os serviços hospitalares mediante dados secundários, resolvemos enfrentar o problema em busca de uma solução.

Apesar de existirem listas de correspondência de códigos CID-9 para CID-10, não existe, na prática, um programa que faça a conversão automática dos códigos CID-9 para códigos CID-10. A correspondência manual costuma ser feita para problemas específicos, mas seria

\section{Resumo}

Para fins de análise epidemiológica, os capítulos da CID e as categorias de três dígitos nem sempre são apropriadas, os primeiros por serem muito abrangentes e os segundos por serem muito numerosos. Quando o interesse epidemiológico não recai sobre diagnósticos específicos, propostas de agrupamento intermediárias entre estas duas possibilidades se fazem necessárias. Tais propostas não aparecem com freqüência na literatura. Nós utilizamos uma proposta de agrupamento, que foi baseada no volume de internações, em duas etapas: na coerência clínica interna de cada grupo e nos resultados do ajuste de um modelo de Cox impraticável no nosso caso, uma vez que trabalhamos com todo o espectro de doenças da CID. Dessa maneira, como iríamos proceder aos agrupamentos descritos, optamos por fazer esse processo em paralelo para as CID-9 e 10 , ainda que tenham sido feitas algumas simplificações, como discutiremos a seguir.

Por exemplo, o código 279 da CID-9, que corresponde às imunodeficiências, deveria ser processado da seguinte forma: o código 279.1, que corresponde à "deficiência da imunidade celular", continha os casos de AIDS na CID-9 10 e, portando, deveria ser alocado nas Doenças Infecciosas e Parasitárias no processo de equivalência, enquanto os demais códigos desse grupo (279.2 a 279.9) deveriam ser alocados nas Doenças do Sangue, Órgãos Hematopoiéticos e Transtornos Imunitários. Como nós trabalhamos apenas com categorias de três dígitos, optamos por alocar o código 279 nas Doenças Infecciosas e Parasitárias. Por outro lado, nenhuma grande inconsistência parece ser introduzida nos capítulos Gravidez, Parto e Puerpério e Afecções Originadas no Período Perinatal, como discutido em Laurenti \& Buchalla 11, à exceção do tétano obstétrico e neonatal.

A CID, como toda classificação, não se ajusta de forma ideal a todas as finalidades, mesmo considerando várias alternativas, como o uso dos capítulos ou apenas dos códigos de três dígitos. Por outro lado, a classificação em quatro dígitos é detalhada o suficiente para permitir agregações diversas para atender às necessidades de cada investigação, sem absolutamente limitar ou restringir as possibilidades. Entretanto, raramente vemos as doenças e agravos serem agrupados de forma mais flexível do que os pré-definidos capítulos ou categorias de três dígitos. Esta é a intenção deste artigo: apresentar e discutir formas de uso e agrupamento da CID que mostrem flexibilidade para buscar respostas específicas para perguntas específicas.

multivariado para dados reais. Obtivemos como resultado 26 grupos para adultos e 19 grupos para crianças, entre os quais escolhemos como categoria de referência hérnia para adultos e doença do apêndice para crianças. O agrupamento obtido se mostrou adequado para a análise de reinternações hospitalares em um grande hospital geral brasileiro. Procedimentos semelhantes ao descrito nesta nota de pesquisa podem ser utilizados para a análise de outros problemas de saúde.

Classificação Internacional de Doenças; Readmissão Hospitalar; Diagnóstico 


\section{Colaboradores}

Ambas as autoras participaram da concepção do artigo, da análise dos dados e da elaboração final do texto.

\section{Referências}

1. Organização Mundial da Saúde. Classificação Internacional de Doenças e problemas relacionados à saúde: décima revisão. São Paulo: Centro Colaborador da OMS para Classificação de Doenças em Português; 1993.

2. Organização Mundial da Saúde. Classificação Estatística Internacional de Doenças: manual de lesões e causas de óbito: nona revisão. São Paulo: Centro Brasileiro para Classificação de Doenças em Português; 1979.

3. Laurenti R. Análise da informação em saúde: 18931993, cem anos da Classificação Internacional de Doenças. Rev Saúde Pública 1991; 25:407-17.

4. César CLH, Laurenti R, Buchalla CM, Figueiredo GM, Carvalho WO, Caratin CVS. Uso da Classificação Internacional de Doenças em inquéritos de saúde. Rev Bras Epidemiol 2001; 4:120-30.

5. Ministério da Saúde. Movimento de Autorização de Internação Hospitalar - reduzido 1997 [CDROM]. Brasília: Ministério da Saúde; 1997.

6. Ministério da Saúde. Movimento de Autorização de Internação Hospitalar - reduzido 2001 [CDROM]. Brasília: Ministério da Saúde; 2001.

7. Cox DR. Regression models and life-tables. Journal of the Royal Statistical Society B 1972; 34:187201.

8. Castro MSM, Carvalho MS, Travassos C. Factors associated with readmission to a general hospital in Brazil. Cad Saúde Pública; no prelo.

9. Ihaka R, Gentleman R. R: a language for data analysis and graphics. Journal of Computational and Graphical Statistics 1996; 5:299-314.

10. Buchalla CM, Laurenti R, Ribeiro AF, Nitrini DRN, Guarnieri CE, Carnaúba EL, et al. Avaliação do uso da Classificação Internacional de Doenças para codificar a síndrome de imunodeficiência adquirida. Rev Saúde Pública 1996; 30:479-82.

11. Laurenti R, Buchalla CM. Maternal and child health indicators: implications of the tenth revision of the International Classification of Dis eases. Rev Panam Salud Publica 1997; 2:13-7.

Recebido em 27/Abr/2004

Versão final reapresentada em 18/Ago/2004

Aprovado em 27/Set/2004 